\title{
Randomized clinical trial: Group counseling based on tinnitus retraining therapy
}

\author{
James A. Henry, PhD; ${ }^{1-2 *}$ Carl Loovis, PhD; ${ }^{3}$ Melissa Montero, BS; ${ }^{1,3}$ Christine Kaelin, MBA; ${ }^{1}$ Kathryn-Anne \\ Anselmi, MEd; ${ }^{3}$ Rebecca Coombs, MS; ${ }^{3}$ June Hensley, MA; ${ }^{3}$ Kenneth E. James, PhD ${ }^{1,4}$ \\ ${ }^{1}$ Department of Veterans Affairs (VA) Rehabilitation Research and Development Service National Center for Rehabili- \\ tative Auditory Research, VA Medical Center, Portland, OR; ${ }^{2}$ Department of Otolaryngology/Head and Neck Surgery, \\ Oregon Health and Science University (OHSU), Portland, OR; ${ }^{3}$ VA Audiology Clinic, VA Puget Sound Health Care \\ System, Seattle, WA; ${ }^{4}$ Department of Public Health and Preventive Medicine, OHSU, Portland, OR
}

\begin{abstract}
The main component of tinnitus retraining therapy (TRT) is structured counseling. We conducted a randomized clinical trial to test the hypothesis that group educational counseling based on TRT principles would effectively treat veterans who have clinically significant tinnitus. Veterans with clinically significant tinnitus were randomized into one of three groups: educational counseling, traditional support, and no treatment. Subjects in the first two groups attended four $1.5 \mathrm{~h}$ group sessions each week. All subjects completed outcome questionnaires at baseline and at 1, 6, and 12 mo. A total of 269 subjects participated: 94 in the educational counseling group, 84 in the traditional support group, and 91 in the no-treatment group. Statistical analyses showed that educational counseling provided significantly more benefit than either traditional support or no treatment, as measured by the Tinnitus Severity Index. Results suggest that group educational counseling can significantly benefit many tinnitus patients and could be integral to a "progressive intervention" approach to tinnitus clinical management.
\end{abstract}

Key words: auditory, clinical trial, educational counseling, hearing disorders, intervention, outcomes, rehabilitation, tinnitus, tinnitus retraining therapy, Tinnitus Severity Index.

\section{INTRODUCTION}

The Department of Veterans Affairs (VA) operates 157 hospitals and 862 community-based outpatient clin- ics to provide healthcare to more than 5 million of the nation's 25 million veterans (http://www.va.gov/JOBS/ VA In Depth/todays va.asp). A major healthcare problem for veterans is "ringing in the ears," i.e., tinnitus. An estimated 3 to 4 million veterans have tinnitus, with up to 1 million needing clinical intervention [1]. The VA regards tinnitus as one of many disabling conditions that veterans can claim to be caused by their military service. Approved claims result in monthly compensation and access to VA clinical services. Veterans are receiving tinnitus disability awards at a rapidly increasing rate. As of September 30, 2006, more than 395,000 veterans were service-connected for tinnitus - an increase of more than 55,000 veterans in 1 year (VA Office of Policy, Planning, and Preparedness, http://www1.va.gov/op3). Despite the

Abbreviations: ANOVA $=$ analysis of variance, $\mathrm{OHSU}=$ Oregon Health and Science University, PVAMC = Portland Department of Veterans Affairs Medical Center, SD = standard deviation, THI = Tinnitus Handicap Inventory, TRT = tinnitus retraining therapy, TSI $=$ Tinnitus Severity Index, VA = Department of Veterans Affairs, VAMC = VA medical center, VAPSHCS = VA Puget Sound Health Care System.

* Address all correspondence to James A. Henry, PhD; VA National Center for Rehabilitative Auditory Research, PO Box 1034, Portland, OR 97207; 503-220-8262, ext 57466; fax: 503-402-2955. Email: james.henry@med.va.gov

DOI: 10.1682/JRRD.2006.02.0018 
breadth of this problem, clinical services for tinnitus are either inadequate or nonexistent at most VA medical centers (VAMCs).

The need to add tinnitus clinical services to VAMCs is readily apparent. The addition of new VA services, however, requires research evidence [2-3]. Furthermore, any new clinical services will affect already strained budgets; thus, the implementation of tinnitus services must be cost-effective. Our objective in designing this study was to develop and evaluate a treatment for tinnitus that would be highly efficient for widespread implementation at VAMCs.

About 80 percent of people who experience chronic tinnitus are not particularly concerned about it [4-5]. The remaining 20 percent have "clinically significant" tinnitus; i.e., they are bothered by it to the degree that clinical intervention is necessary. Of those patients requiring clinical care, only a small proportion have "very severe problems" [6]. Most require education about tinnitus and effective coping strategies. Patient education is an essential component of all health practices [7], and an educational approach has been used in the management of tinnitus for many years [8]. Group education provides a more cost-effective alternative to individual education, and research evidence indicates that group education can be as or even more effective than individual education [9-10]. Only one controlled study has evaluated the effectiveness of group education for tinnitus [11]. In that study, group education was the control group and no overall benefit to patients in this group was found. In contrast, a well-known tinnitus clinic presently uses group education as part of its tinnitus management protocol. This clinic reported that the majority of a sample of 52 patients had reduced self-perceived tinnitus impairment following a single educational session and found the session beneficial [12].

At the Portland VAMC (PVAMC), a tinnitus support/ education group for veterans has been conducted regularly since 1999. The group focuses on the provision of useful information for reducing tinnitus impact, and the information received at these meetings has successfully met many veterans' needs. Based on this experience and the literature supporting the effectiveness of group education across a wide range of medical disciplines [9-10], group education for basic tinnitus intervention is a potentially efficient method for VA-wide implementation. The present study was designed around this premise. The specific education provided in the group meetings was an adaptation and expansion of the educational counseling protocol for tinnitus retraining therapy (TRT) [13]. The TRT educational counseling protocol was selected because, at the time, it was the only well-defined educational counseling protocol for tinnitus patients. TRT has also been shown to be an effective treatment when used on an individual basis [14-16].

TRT was developed by Jastreboff and is a clinical implementation of his "neurophysiological model of tinnitus" [16]. The model was developed in the 1980s and formally presented in 1990 [17]. TRT training workshops have been conducted since the mid-1990s, and TRT is currently performed in more than 100 clinics worldwide.

The TRT neurophysiological model describes problematic tinnitus as being caused by an aberrant signal in the auditory nervous system that has been conditioned to activate the limbic and autonomic nervous systems, resulting in emotional reactions and stress [16]. The aberrant signal must undergo reconditioning to be reclassified by brain processing centers as a meaningless, unimportant signal. The reconditioning process is referred to as "retraining" the brain to habituate to the tinnitus signal.

The primary objective of TRT is habituation of the negative reactions associated with the tinnitus [16]. This objective is achieved through structured educational counseling. The second objective, which can be successful only if the first objective is successful, is habituation of tinnitus from conscious perception. The second objective involves "sound therapy," which is the use of constant low-level sound to reduce the "detectability" of tinnitus at subconscious levels. The state of reduced detectability of the tinnitus signal must be maintained for a sufficient period of time to retrain the tinnitus signalprocessing mechanism.

For sound therapy, patients with more severe tinnitus are normally fitted with ear-level wearable devices such as sound generators, hearing aids, or instruments that combine a sound generator and hearing aid in the same casing. Patients wear the devices during all waking hours for at least 1 year and follow a very specific use protocol [16]. These patients also attend clinical appointments on a prescribed schedule; the TRT counseling is administered at every appointment. TRT does not necessarily involve the use of ear-level devices. Patients with less severe tinnitus receive only counseling to the degree necessary. Even some patients with more severe tinnitus (estimated at $10 \%-30 \%$ of the most severe clinical cases) can benefit from counseling alone by effectively starting the habituation 
process [13]. Thus, the most important aspect of TRT is educational counseling. Jastreboff stated, "Proper counseling, including a clear explanation of the physiology of hearing and present knowledge about tinnitus generation and perception, is the first and essential part of any treatment” [18, p. 85]. Regardless of whether ear-level devices are used with TRT, the counseling always includes detailed advice on how patients can add low-level, nonannoying sound to every environment.

We hypothesized that we could provide effective and efficient treatment to veterans with clinically significant tinnitus by using group educational counseling adapted from the structured TRT counseling protocol. We conducted a randomized clinical trial to test this hypothesis.

\section{MATERIALS AND METHODS}

\section{Study Site}

The VA Puget Sound Health Care System (VAPSHCS) in Washington State was selected as the data collection site because of its proximity to the PVAMC and because it had never had a tinnitus clinical program. Thus, the likelihood of achieving the required number of "untainted" subjects through the VAPSHCS was higher than from the PVAMC, which has had a long-term tinnitus clinical program in addition to numerous tinnitus research projects. The VAPSHCS includes the American Lake (Tacoma) and Seattle VAMCs.

\section{Subject Recruitment}

Subjects were recruited from the Seattle/Tacoma area via local newspaper and radio advertisements and flyers posted at the Seattle and American Lake VAMCs. Approximately 750 veterans responded to the advertisements by telephoning the project coordinator, who asked them four scripted questions: (1) Do you have tinnitus that is constant? (2) Does tinnitus affect your sleep? (3) Does tinnitus affect your reading or concentration? and (4) On a scale of 1 to 10 , how much has tinnitus annoyed you in the last month ( 1 being not at all, 10 being as much as you can imagine)? Callers were considered potential candidates, regardless of age or medical condition, if they (1) had clinically significant tinnitus, i.e., if their tinnitus was sufficiently bothersome to warrant intervention; (2) were willing and able to complete all study requirements; and (3) attended an open house, where they received further information about the study. At the open house, interested candidates were asked to sign informed consent forms and complete baseline ques- tionnaires. The use of human subjects for this research was approved by the Institutional Review Board Committee at the University of Washington, which reviews human subject research at the Seattle and American Lake VAMCs.

Of the 750 callers, 549 were invited to six open houses held over 9 months. Of the 549 invited veterans, 373 (68\%) attended. Of these, 294 (79\%) agreed to participate and were enrolled; 25 participated in a pilot phase for testing the education intervention and study procedures and 269 participated in the randomized phase.

\section{Subjects}

Using an electronically generated randomization schedule, we randomly assigned the 269 veterans to one of three groups: educational counseling $(n=94)$, traditional support $(n=84)$, and no-treatment $(n=91)$. Age and sex information for all subjects is provided in Table 1.

We used the baseline questionnaires to obtain demographic information, tinnitus history, hearing history, noise-exposure history, medical history, and descriptions of the subjects' tinnitus. Selected questions from the baseline questionnaires are shown in Table 2, along with the numbers of randomized subjects who provided different response choices. For each question, responses were distributed in similar proportions across the three groups. Combining the data (totals shown in Table 2), the responses can be summarized as follows:

1. About the same number of subjects were employed $(44.4 \%)$ as retired (43.2\%).

2. Most subjects had either attended (33.8\%) or completed (51.9\%) college.

3. Subjects generally (85.8\%) rated their overall health as "good" or better.

4. Most subjects (93.0\%) reported "difficulty hearing” at least "sometimes."

5. Most subjects (83.5\%) thought that their tinnitus "made it more difficult to hear" at least "sometimes."

Table 1.

Age and sex of randomized subjects.

\begin{tabular}{lcccccc}
\hline \multirow{2}{*}{ Group } & \multirow{2}{*}{$\boldsymbol{n}$} & \multicolumn{2}{c}{ Age (yr) } & & \multicolumn{2}{c}{ Sex } \\
\cline { 3 - 4 } & & Mean \pm SD & Range & & M & F \\
\hline Educational Counseling & 94 & $62.1 \pm 8.9$ & $42-83$ & & 91 & 3 \\
Traditional Support & 84 & $60.8 \pm 9.5$ & $33-80$ & & 81 & 3 \\
No-Treatment & 91 & $62.0 \pm 11.3$ & $35-86$ & & 88 & 3 \\
\hline Total & 269 & $61.6 \pm 9.9$ & $33-86$ & & 260 & 9 \\
\hline F = female, M = male, SD = standard deviation. & & & \\
\hline
\end{tabular}


JRRD, Volume 44, Number 1, 2007

Table 2.

Responses to study questions by subjects in education, support, and no-treatment (NT) groups. Data from baseline questionnaires.

\begin{tabular}{|c|c|c|c|c|}
\hline \multirow{2}{*}{ Question/Response Choices } & \multicolumn{4}{|c|}{ Response (No. of Subjects) } \\
\hline & Educational Counseling & Traditional Support & NT & Total \\
\hline \multicolumn{5}{|l|}{ “Are you currently employed?” } \\
\hline$n$ & 93 & 84 & 89 & 266 \\
\hline Full-Time & 34 & 36 & 34 & 104 \\
\hline Part-Time & 7 & 3 & 4 & 14 \\
\hline Retired & 39 & 36 & 40 & 115 \\
\hline Looking for Work & 2 & 3 & 5 & 10 \\
\hline Unemployed Due to Health & 9 & 4 & 3 & 16 \\
\hline Other & 2 & 2 & 3 & 7 \\
\hline \multicolumn{5}{|c|}{ "What is the highest grade in school you completed?" } \\
\hline$n$ & 94 & 83 & 89 & 266 \\
\hline$\leq 8$ th Grade & 0 & 0 & 0 & 0 \\
\hline Some High School & 0 & 0 & 0 & 0 \\
\hline High School & 10 & 5 & 6 & 21 \\
\hline Vocational & 9 & 3 & 5 & 17 \\
\hline Some College & 33 & 27 & 30 & 90 \\
\hline College & 42 & 48 & 48 & 138 \\
\hline \multicolumn{5}{|l|}{ "How is your health, comparatively?” } \\
\hline$n$ & 94 & 84 & 89 & 267 \\
\hline Excellent & 15 & 10 & 20 & 45 \\
\hline Very Good & 31 & 39 & 26 & 96 \\
\hline Good & 36 & 22 & 30 & 88 \\
\hline Fair & 7 & 10 & 12 & 29 \\
\hline Poor & 5 & 3 & 1 & 9 \\
\hline \multicolumn{5}{|c|}{ “Do you experience difficulty hearing?” } \\
\hline$n$ & 94 & 84 & 91 & 269 \\
\hline Never & 1 & 3 & 5 & 9 \\
\hline Rarely & 1 & 4 & 5 & 10 \\
\hline Sometimes & 30 & 33 & 25 & 88 \\
\hline Usually & 25 & 20 & 29 & 74 \\
\hline Always & 37 & 24 & 27 & 88 \\
\hline \multicolumn{5}{|c|}{ "Does your tinnitus make it more difficult for you to hear?" } \\
\hline$n$ & 93 & 80 & 87 & 260 \\
\hline Never & 0 & 2 & 3 & 5 \\
\hline Rarely & 6 & 6 & 5 & 17 \\
\hline Sometimes & 22 & 26 & 32 & 80 \\
\hline Usually & 26 & 21 & 26 & 73 \\
\hline Always & 28 & 18 & 18 & 64 \\
\hline Unsure & 11 & 7 & 3 & 21 \\
\hline \multicolumn{5}{|l|}{ "How long have you had tinnitus?" } \\
\hline$n$ & 94 & 84 & 91 & 269 \\
\hline$<1 \mathrm{yr}$ & 4 & 2 & 3 & 9 \\
\hline $1-2 \mathrm{yr}$ & 3 & 1 & 3 & 7 \\
\hline $3-5 \mathrm{yr}$ & 8 & 6 & 7 & 21 \\
\hline $6-10 \mathrm{yr}$ & 11 & 11 & 17 & 39 \\
\hline $10-20 \mathrm{yr}$ & 28 & 16 & 18 & 62 \\
\hline$>20 \mathrm{yr}$ & 37 & 41 & 36 & 114 \\
\hline Unsure & 3 & 7 & 7 & 17 \\
\hline \multicolumn{5}{|l|}{ "What is the location of your tinnitus?" } \\
\hline$n$ & 94 & 83 & 91 & 268 \\
\hline Left Ear Only & 6 & 4 & 10 & 20 \\
\hline Right Ear Only & 2 & 2 & 11 & 15 \\
\hline Both Ears & 76 & 65 & 61 & 203 \\
\hline
\end{tabular}


Table 2. (Continued)

Responses to study questions by subjects in education, support, and no-treatment (NT) groups. Data from baseline questionnaires.

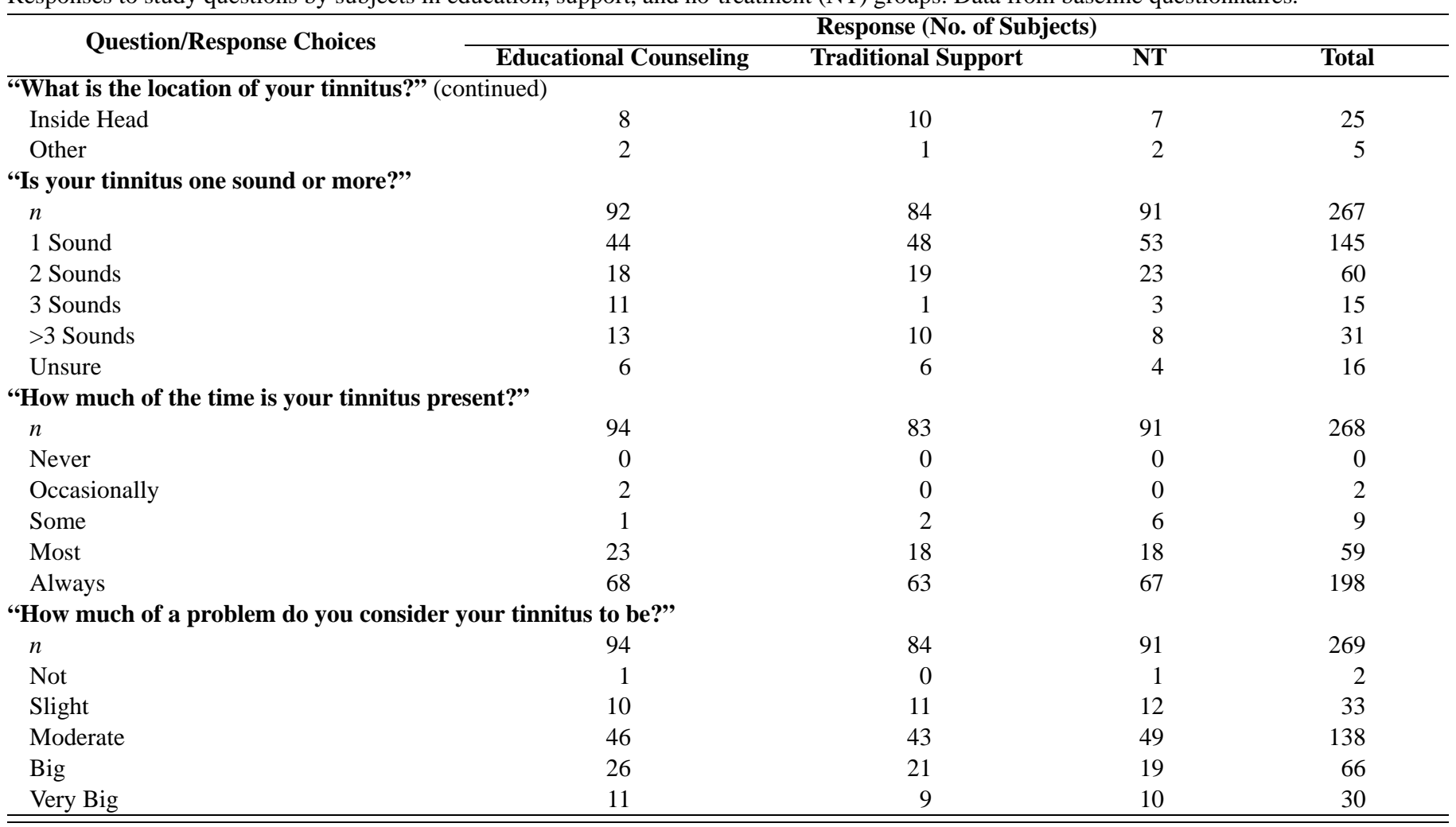

6. Most subjects (87.7\%) had experienced tinnitus for at least 3 years.

7. Most subjects (75.7\%) localized their tinnitus to "both ears."

8. More than half of subjects (54.3\%) perceived their tinnitus as a single sound, while most of the remainder perceived two or more sounds.

9. Almost all subjects (95.9\%) reported that their tinnitus was present "most of the time" or "always."

10. Most subjects (87\%) considered their tinnitus to be at least a "moderate" problem.

\section{Procedures}

Subjects in the educational counseling group attended four weekly sessions. Each session lasted 1.5 hours, including 15 minutes for general discussion. One of three study audiologists conducted the educational presentations for each cohort of subjects. The topics covered during the four sessions were-

\section{- Session 1}

- Introduction and description of terms.
- General overview of TRT.

- Discussion of what patients "hear" as tinnitus.

- Explanation of sound waves.

- Basic anatomy and physiology of ear and auditory pathways.

- How acoustic vibrations are converted to nerve impulses.

- Neural pattern recognition.

- Perception of sound in auditory cortex.

- "Selective" listening.

\section{- Session 2}

- Brief review of Session 1 material.

- Nature of tinnitus as "phantom" auditory sensation.

- Why tinnitus becomes a problem.

- Addressing misconception that tinnitus causes hearing difficulties.

- Explanation of audiogram, with examples of hearing loss.

- Explanation of habituation as goal of TRT.

- Subconscious processing of auditory stimuli. 
- "Filtering" and "blocking" auditory stimuli from reaching consciousness.

- Why tinnitus enters consciousness.

\section{- Session 3}

- Brief review of Session 2 material.

- Why monotonous sounds are habituated naturally.

- Why tinnitus may not be habituated.

- "Vicious circle" of listening and reacting.

- Heller and Bergman's 1953 experiment, and its relevance to tinnitus perception.

- Explanation of TRT "sound therapy" with examples.

- Why "maskers" are not used with TRT.

- Use of ear-level sound generators for TRT.

- Overview of TRT "neurophysiological model."

- Detailed description of cochlea and hair cells.

- Detailed description of auditory nerve.

- Description of otoacoustic emissions and cochlear amplifier.

- Brief description of hyperacusis.

\section{- Session 4}

- Brief review of Session 3 material.

- Detailed explanation of hyperacusis.

- Description of auditory gain and how it is controlled.

- Description of "neural networks."

- "Discordant damage" theory of tinnitus generation.

- Further description of auditory processing - related to neurophysiological model.

- Relation of auditory cortex to neurophysiological model.

- Identification of and assigning meaning to auditory signals.

- Neurophysiological model—nonauditory systems.

- Description of limbic system.

- Explanation of emotions based on neurophysiological model.

- Description of autonomic nervous system.

- Feedback loops.

- Stress and tinnitus.

- Brain plasticity.

- Final advice.

Traditional-support subjects attended four weekly 1.5-hour discussion-type group sessions. Sessions were moderated by the project coordinator. No education was provided in the support group. The no-treatment group did not receive any study intervention.
Subjects in the educational counseling and traditional support groups attended their first session within 4 to 8 weeks of completing the baseline questionnaires at an open house. Follow-up questionnaires were administered by mail and completed by subjects in the educational counseling and traditional support groups 1, 6, and 12 months after their last group session. The no-treatment subjects completed the follow-up questionnaires 1,6 , and 12 months after their enrollment at an open house.

The 12-item Tinnitus Severity Index (TSI) was the primary instrument for assessing outcomes (Appendix, available online only at http://www.rehab.research.va.gov/). The TSI ranges from 0 to 48 and has a Cronbach $\alpha$ of 0.92 [19] and test-retest reliability of 0.88 [20]. The TSI was developed at the Oregon Health and Science University (OHSU) Tinnitus Clinic, where it has been used for many years with thousands of patients [21]. Based on data from OHSU Tinnitus Clinic patients, the TSI has a normal distribution with a mean of 26.8 and standard deviation (SD) of 9.8. In a separate clinical trial, we used the TSI along with two other well-accepted tinnitus instruments, the Tinnitus Handicap Inventory (THI) [22-23] and the Tinnitus Handicap Questionnaire [24]. Results were comparable between the TSI and these instruments [14-15], which further validates use of the TSI for assessing treatment outcomes. Subjects in that trial, which involved 18 months of individualized treatment, were screened to ensure that their tinnitus was sufficiently severe to warrant such intensive treatment. Their mean \pm SD TSI score was $28.8 \pm 8.7$.

\section{Data Analysis}

For each of the three groups, we calculated paired $t$ tests to determine if the mean TSI scores differed significantly between baseline and the three follow-up time points (1, 6, and 12 months). We performed one-way analyses of variance (ANOVAs) on the baseline adjusted means at the three time points to determine if the time effect of each group varied across the groups. The baseline to 12-month change in mean TSI score was the primary study outcome. In addition, we calculated effect sizes to standardize the outcome data [25].

\section{RESULTS}

Baseline mean TSI scores were 24.8 for the educational counseling group, 22.9 for the traditional support group, and 22.3 for the no-treatment group. An ANOVA 
determined that these means were not significantly different $(p>0.05)$. Table 3 shows the numbers of subjects in each group that completed the TSI at each time point. Paired $t$-tests were used to evaluate changes in mean TSI scores within each group. Significance was set at $p=$ 0.05 and corrected for multiple $t$-tests ( $p=0.05 / 9=$ 0.006; three groups $\times$ three time-point comparisons). Data for the baseline to 6-month and 12-month comparisons are shown in Tables 4 and 5, respectively. The educational counseling group showed a significant reduction in mean TSI score from baseline to 6 months $(p<0.001)$ and baseline to 12 months $(p<0.001)$. The changes in mean TSI score from baseline to 6 months and baseline to 12 months were not significant for the traditional support and no-treatment groups.

We calculated one-way ANOVAs to determine if the baseline-adjusted means significantly differed between the three groups; i.e., we compared mean difference scores. The null hypothesis that all three groups produced equal reductions in TSI score failed to be rejected for the 1-month difference $(p=0.062)$ but was rejected for the 6-month $(p=0.003)$ and 12-month differences ( $p=$ $0.027)$. Since the ANOVAs for the 6 - and 12-month differences were significant, specific treatment contrasts were calculated. At 6 months, a significant difference existed between the educational counseling and traditional support groups ( $p=0.015$ ) and between the educational counseling and no-treatment groups $(p=0.001)$ but not between the traditional support and no-treatment groups ( $p=0.470)$. At 12 months, a significant difference existed between the educational counseling and traditional support groups ( $p=0.033$ ) and between the educational counseling and no-treatment groups $(p=0.013)$ but not between the traditional support and no-treatment groups ( $p=0.824)$. The $p$-values for these differences are unadjusted for multiple comparisons.

Effect sizes indicate the magnitude of the "real" treatment effect, and their use standardizes outcome data

Table 3.

Number of subjects who completed Tinnitus Severity Index (TSI) at each outcome point.

\begin{tabular}{lcccc}
\hline \multicolumn{1}{c}{ Group } & Baseline & 1 Mo & 6 Mo & 12 Mo \\
\hline Educational Counseling & 94 & 72 & 67 & 68 \\
Traditional Support & 84 & 62 & 60 & 61 \\
No-Treatment & $90^{*}$ & 83 & 73 & 76 \\
\hline Total & 268 & 217 & 200 & 205 \\
\hline *One no-treatment subject did not complete baseline TSI. & \\
\hline \hline
\end{tabular}

across different instruments [25]. We calculated effect sizes for the TSI outcomes at 6 and 12 months, which are shown in Tables 4 and 5, respectively. Cohen suggests that, as a general rule of thumb, effect sizes of $0.20,0.50$, and 0.80 are considered small, medium, and large, respectively [26]. The effect sizes for the educational counseling group were 0.59 at 6 months and 0.45 at 12 months, while the effect sizes for the traditional support and notreatment groups were 0.11 or less at 6 and 12 months.

\section{DISCUSSION}

We conducted this randomized clinical trial to evaluate the efficacy of TRT-based group educational counseling for the treatment of individuals with clinically significant tinnitus. Outcomes were evaluated at baseline, 1 month, 6 months, and 12 months for the educational counseling group, a traditional support group, and a notreatment group. Statistical analyses revealed that group educational counseling provided significant benefit based on mean outcome scores. This benefit was sustained over the 12-month outcome period. The traditional support and no-treatment groups did not show significant improvement at any outcome point.

This study was rigorously controlled with respect to key variables: (1) both the educational counseling and traditional support groups had multiple cohorts of subjects, (2) three clinicians performed the educational counseling sessions, (3) the traditional support group provided equal contact-time but no education, and (4) the no-treatment group documented that no changes occurred when no intervention was provided. These observations support the major study outcome, i.e., that TRT-based group educational counseling had an overall beneficial effect on individuals with clinically significant tinnitus.

In the peer-reviewed literature, only one controlled study has evaluated group education as a treatment for tinnitus [11]. Sixty subjects (mean age 64.6 years) were randomized into one of three groups: combined cognitive/ education therapy, education alone, or no-treatment. The two treatments were administered to groups of five to seven subjects during six weekly 90 -minute sessions. Outcomes were assessed at baseline, immediate posttreatment, and 12 months posttreatment. The same education was provided to both groups and covered the auditory system and various aspects of tinnitus. The cognitive/education group additionally received instruction in cognitive coping skills. 
JRRD, Volume 44, Number 1, 2007

Table 4.

Paired $t$-test results and effect sizes for Tinnitus Severity Index (TSI) scores (mean \pm standard deviation) by group, between baseline and 6 months.

\begin{tabular}{llllrrrr}
\hline \multicolumn{1}{c}{ Group } & $\boldsymbol{n}^{*}$ & Baseline & \multicolumn{1}{c}{$\mathbf{6 ~ M o}$} & Difference & $\boldsymbol{t}$-Test & $\boldsymbol{p}$-Value & Effect Size \\
\hline Educational Counseling & 67 & $24.9 \pm 8.7$ & $21.6 \pm 10.3$ & $-3.2 \pm 5.4$ & -4.95 & $<0.001$ & 0.59 \\
Traditional Support & 60 & $23.6 \pm 10.4$ & $23.0 \pm 9.6$ & $-0.7 \pm 6.7$ & -0.79 & 0.43 & 0.10 \\
No-Treatment & 73 & $22.0 \pm 8.4$ & $22.0 \pm 9.3$ & $0.0 \pm 5.3$ & -0.09 & 0.93 & 0.00 \\
\hline
\end{tabular}

*Data for 200 total subjects who completed TSI at both time points.

${ }^{\dagger}$ Adjusted for multiple comparisons.

Table 5.

Paired $t$-test results and effect sizes for Tinnitus Severity Index (TSI) scores (mean \pm standard deviation) by group, between baseline and 12 months.

\begin{tabular}{lllllccc}
\hline \multicolumn{1}{c}{ Group } & $\boldsymbol{n}^{*}$ & Baseline & \multicolumn{1}{c}{ 12 Mo } & Difference & $\boldsymbol{t}_{\text {-Test }}$ & $\boldsymbol{p}$-Value & Effect Size \\
\hline Educational Counseling & 68 & $25.1 \pm 8.8$ & $22.1 \pm 11.0$ & $-3.0 \pm 6.6$ & -3.70 & $<0.001$ & 0.45 \\
Traditional Support & 61 & $23.6 \pm 10.3$ & $22.9 \pm 9.3$ & $-0.7 \pm 6.4$ & -0.84 & 0.40 & 0.11 \\
No-Treatment & 75 & $22.1 \pm 8.5$ & $21.6 \pm 8.9$ & $-0.5 \pm 4.9$ & -0.81 & 0.42 & 0.10 \\
\hline
\end{tabular}

* Data for 204 total subjects who completed TSI at both time points.

${ }^{\dagger}$ Adjusted for multiple comparisons.

Significant improvement in outcomes was observed for the cognitive/education group immediately posttreatment, but these gains were lost at 12 months. The education and notreatment groups showed no improvement at either the immediate or 12-month posttreatment outcome points. In contrast, the present study showed improvement in the educational counseling group that was sustained for 12 months. This durable improvement may have been due to the TRTspecific counseling that was used.

Our findings support a proposed model of tinnitus services that use a "progressive intervention" approach [20]. This approach involves hierarchical levels of service that provide treatment only to the degree needed by individual patients. In this model, screening for clinically significant tinnitus is Level 1 of five levels of service. Effective screening separates patients who require clinical management from those who do not [27]. Those requiring clinical management can receive cost-effective treatment with the group education approach (Level 2). After receiving group education, patients decide whether they need further services [12]. If so, they should be scheduled for a tinnitus intake evaluation (Level 3), which includes questionnaires, audiological testing, and a tinnitus psychoacoustic assessment [27]. The evaluation determines the need for ongoing treatment (Level 4). Level 5 refers to the rare need for treatment beyond 1 to 2 years. At all levels, patients should be referred appropriately for medical or mental healthcare.

One major tinnitus clinic uses a variation of the progressive management approach, with group education provided following a basic audiological assessment [12]. Fifty-two patients who received group education in that program were studied to (1) evaluate changes in their perceived impairment following participation in the group education session, (2) evaluate their perceived benefit from attending the session, and (3) determine their willingness to pay for such a session. The THI was completed before the session and 30 days later to assess changes in perceived tinnitus impairment [22-23]. Results revealed a clinically significant improvement for the group of patients based on the THI. Attending the session was considered beneficial by 69 percent of the patients, and 43 percent planned to return for an individual tinnitus assessment, which was the next level of service in this program. In summary, most patients had a reduction in self-perceived tinnitus impairment following the group education session and found the session beneficial. On average, the patients indicated willingness to pay for the session at a rate that was similar to fee-for-service.

An important consideration, especially for the VA or other clinics that operate on very tight time schedules, is per-patient contact time. Subjects in the educational counseling group of the present study attended four 1.5-hour sessions. Based on an average of 20 subjects attending each session, the total per-patient contact time was about 18 minutes. Individualized counseling can require up to 1 hour or more for a single session. In addition, group education involves classroom-style instruction only, obviating individual appointments, audiological testing, and the selecting and of fitting ear-level devices. Some patients, of 
course, require these and other clinical services. If a clinical assessment is not conducted before the group session, then the counseling should include (1) a discussion of symptoms that would suggest the need for referralusually to an otolaryngologist or mental health professional, (2) an explanation of how hearing loss can be blamed inappropriately on tinnitus and how to receive a hearing evaluation if this is a concern, and (3) an explanation that further treatment is available and how to receive it.

Of note, almost 84 percent of the subjects in this study responded that they experienced hearing difficulty at least "sometimes" and 88 percent indicated that their tinnitus made hearing more difficult at least "sometimes" (Table 2). Tinnitus patients commonly have hearing loss, and they often believe that their tinnitus is the root cause of their hearing difficulties. However, the belief that tinnitus interferes with hearing sensitivity is usually a misconception [6,28-30]. Thus, while education can help a patient understand and cope more effectively with a tinnitus problem, no amount of education can correct a hearing problem. Tinnitus patients who experience hearing difficulties require a hearing assessment and potentially hearing aids [9]. The results of the educational counseling in the current study were thus limited by the inability to correct any perceived hearing deficits. Because a high percentage of subjects indicated that hearing was a problem, this limitation was likely a significant factor in reducing the effectiveness of the educational intervention.

This project was well received by the participating audiologists at the Seattle and American Lake VAMCs, where no tinnitus management program previously existed. These clinics have now instituted a clinical program for their tinnitus patients that uses a similar groupeducation format. Instead of conducting four educational sessions, they have condensed the structured information into a single session. The fact that these patients have not been returning to the clinic indicates that the group session in some way resolved their concerns.

The next step in this continuing research is modification of the group education program to optimize outcomes of treatment. Such optimization may be best accomplished by maximization of patient retention of the counseling information. Numerous studies have shown that patients' compliance with treatment, reduction in distress, and speed and quality of recovery are directly related to their comprehension and recall of medical advice [31]. Methods of improving retention can include (1) enhancing learning through repetition (repetition of core information during each session while varying supplementary information), (2) increasing the length of sessions, (3) including a structured patient-centered component that addresses individual concerns, (4) providing take-home information, (5) enhancing presentation graphics to improve recall, and (6) spacing sessions over longer intervals to increase long-term recall. These strategies should enhance clinical outcomes relative to the results reported for this study.

\section{CONCLUSIONS}

Basic counseling information for tinnitus patients can be provided in a group setting, which offers several advantages. Clinical resources are maximized when patients into are consolidated groups. The education empowers patients by informing them about strategies for self-management and the treatment options available. In addition, patients benefit from talking with others who experience the same problem. Several tinnitus clinics are currently offering structured educational meetings on a regular basis. The present study indicates that the group education approach can significantly benefit tinnitus patients who experience different levels of tinnitus severity. Further research must determine the optimal format and parameters for conducting these groups.

\section{ACKNOWLEDGMENTS}

Special thanks to Stephen A. Fausti, PhD, for his ongoing support, and Hal Pashler, PhD, and Kimberly Owens, BS, for specific contributions.

This material was based on work supported by the Veterans Health Administration and the VA Rehabilitation Research and Development Service (grant C2760R).

The authors have declared that no competing interests exist.

\section{REFERENCES}

1. Henry JA, Schechter MA, Regelein RT, Dennis KC. Veterans and tinnitus. In: Snow JB Jr, editor. Tinnitus: Theory and management. Lewiston (NY): BC Decker, Inc; 2004. p. 337-55.

2. Darby M. Clinical practice guidelines. Boston (MA): Management decision and research center. Washington (DC): 
Department of Veterans Affairs Health Services Research and Development Service; 1998.

3. Feussner JR. Clinical research in the Department of Veterans Affairs: Using research to improve patient outcomes. J Investig Med. 1998;46(6):264-67. [PMID: 9737085]

4. Jastreboff PJ, Hazell JWP. Treatment of tinnitus based on a neurophysiological model. In: Vernon JA, editor. Tinnitus treatment and relief. Needham Heights (MA): Allyn \& Bacon; 1998. p. 201-17.

5. Davis A, Refaie AE. Epidemiology of tinnitus. In: Tyler RS, editor. Tinnitus handbook. San Diego (CA): Singular Publishing Group; 2000. p. 1-24.

6. Dobie RA. Overview: Suffering from tinnitus. In: Snow JB Jr, editor. Tinnitus: Theory and management. Lewiston (NY): BC Decker, Inc; 2004. p. 1-7.

7. Redman BK. The practice of patient education. St. Louis (MO): Mosby; 1997.

8. Wilson PH, Henry JL, Andersson G, Hallam RS, Lindberg P. A critical analysis of directive counselling as a component of tinnitus retraining therapy. Br J Audiol. 1998;32(5):273-87. [PMID: 9845025]

9. Mensing CR, Norris SL. Group education in diabetes: Effectiveness and implementation. Diabetes Spectrum. 2003; 16(2):96-103.

10. Wilson SR. Individual versus group education: Is one better? Patient Educ Couns. 1997;32(1 Suppl):S67-75. [PMID: 9516762]

11. Henry JL, Wilson PH. The psychological management of tinnitus: Comparison of a combined cognitive educational program, education alone and a waiting-list control. Int Tinnitus J. 1996;2:9-20. [PMID: 10753339]

12. Newman CW, Sandridge SA. Incorporating group and individual sessions into a tinnitus management clinic. In: Tyler RS, editor. Tinnitus treatment: Clinical protocols. New York (NY): Thieme Medical Publishers; 2005. p. 187-97.

13. Jastreboff PJ. Tinnitus habituation therapy (THT) and tinnitus retraining therapy (TRT). In: Tyler RS, editor. Tinnitus handbook. San Diego (CA): Singular Publishing Group; 2000. p. 357-76.

14. Henry JA, Schechter MA, Zaugg TL, Griest SE, Jastreboff PJ, Vernon JA, Kaelin C, Meikle MB, Lyons KS, Stewart BJ. Clinical trial to compare tinnitus masking and tinnitus retraining therapy. Acta Otolaryngol Suppl. 2006;126(556):64-69. [PMID: 17114146]

15. Henry JA, Schechter MA, Zaugg TL, Griest SE, Jastreboff PJ, Vernon JA, Kaelin C, Meikle MB, Lyons KS, Stewart BJ. Outcomes of clinical trial: Tinnitus masking versus tinnitus retraining therapy. J Am Acad Audiol. 2006;17(2):104-32. [PMID: 16640064]

16. Jastreboff PJ, Hazell JW. Tinnitus retraining therapy: Implementing the neurophysiological model. Cambridge (England): Cambridge University Press; 2004.
17. Jastreboff PJ. Phantom auditory perception (tinnitus): Mechanisms of generation and perception. Neurosci Res. 1990;8(4):221-54. [PMID: 2175858]

18. Jastreboff PJ. Tinnitus as a phantom perception: Theories and clinical implications. In: Vernon JA, Moller AR, editors. Mechanisms of tinnitus. Needham Heights (MA): Allyn \& Bacon; 1995. p. 73-93.

19. Meikle MB, Griest SE, Stewart BJ, Press LS. Measuring the negative impact of tinnitus: A brief severity index. In: Ryan A, editor. Midwinter Meeting: Association for Research in Otolaryngology; 1995 Feb 5-9; St. Petersburg Beach, FL. Des Moines (IA): Association for Research in Otolaryngology; 1995. p. 167.

20. Henry JA, Schechter MA, Loovis CL, Zaugg TL, Kaelin C, Montero M. Clinical management of tinnitus using a "progressive intervention” approach. J Rehabil Res Dev. 2005; 42(4 Suppl 2):95-116. [PMID: 16470767]

21. Johnson RM. The masking of tinnitus. In: Vernon JA, editor. Tinnitus treatment and relief. Needham Heights (MA): Allyn \& Bacon; 1998. p. 164-86.

22. Newman CW, Jacobson GP, Spitzer JB. Development of the Tinnitus Handicap Inventory. Arch Otolaryngol Head Neck Surg. 1996;122(2):143-48. [PMID: 8630207]

23. Newman CW, Sandridge SA, Jacobson GP. Psychometric adequacy of the Tinnitus Handicap Inventory (THI) for evaluating treatment outcome. J Am Acad Audiol. 1998;9(22):153-60. [PMID: 9564679]

24. Kuk FK, Tyler RS, Russell D, Jordan H. The psychometric properties of a tinnitus handicap questionnaire. Ear Hear. 1990;11(6):434-45. [PMID: 2073977]

25. Lipsey MW, Aikens LS, West SG. Design sensitivity: Statistical power for experimental research. Newbury Park (CA): Sage Publications, Inc; 1990.

26. Cohen J. Statistical power analysis for the behavioral sciences. Hillsdale (NJ): Lawrence Erlbaum Associates, Inc; 1988.

27. Henry JA, Zaugg TL, Schechter MA. Clinical guide for audiologic tinnitus management I: Assessment. Am J Audiol. 2005;14(1):21-48. [PMID: 16180968]

28. Coles RR. Classification of causes, mechanisms of patient disturbance, and associated counseling. In: Vernon JA, Moller AR, editors. Mechanisms of tinnitus. Needham Heights (MA): Allyn \& Bacon; 1995. p. 11-19.

29. Henry JA, Dennis KC, Schechter MA. General review of tinnitus: Prevalence, mechanisms, effects, and management. J Speech Lang Hear Res. 2005;48(5):1204-35.

30. Zaugg T, Schechter MA, Fausti SA, Henry JA. Difficulties caused by patients' misconceptions that hearing problems are due to tinnitus. In: Patuzzi R, editor. Proceedings of the Seventh International Tinnitus Seminar; 2002 Mar 5-9; Fremantle, Australia. Perth (Australia): Physiology Department, University of Western Australia; 2002. p. 226-28. 
31. Ley P. Psychological studies of doctor-patient communication. In: Rachman S, editor. Contributions to medical psychology. Oxford (England): Pergamon Press; 1977. p. 9-42.
Submitted for publication February 23, 2006. Accepted in revised form October 27, 2006. 